\section{New Toolkits for Positron Emission Tomography}

Positron emission tomography
(PET) is a powerful imaging
technique that uses radiotracers
injected into the body to
look at biology in tissues and
cells, making it an important
tool in biomedical research
and drug development. Dr
Victor Pike, Chief of the PET
Radiopharmaceutical Sciences
Section of the Molecular
Imaging Branch at the National
Institute of Mental Health
in the U.S., has been using
radiochemistry to develop
new radiotracers with not only
long-term potential to improve
existing medical diagnosis and
treatment but also to enhance
the use of PET to look anew
at diseases and biological
processes.

edical imaging is all about

finding ways to peer inside our and problems that cannot be seen depend heavily on the help of medica imaging devices and their inventive ways of visualising internal organs, tissues and biological processes.

Most types of medical imaging devices invisible to the human eye. Probably the most widely used kind of light are $X$-rays, an example of light with a very short wavelength that is very efficienty absorbed by dense tissue like bone. This strong absorption means that $X$-rays can give very high contrast images of the structure of the bone and fractures.

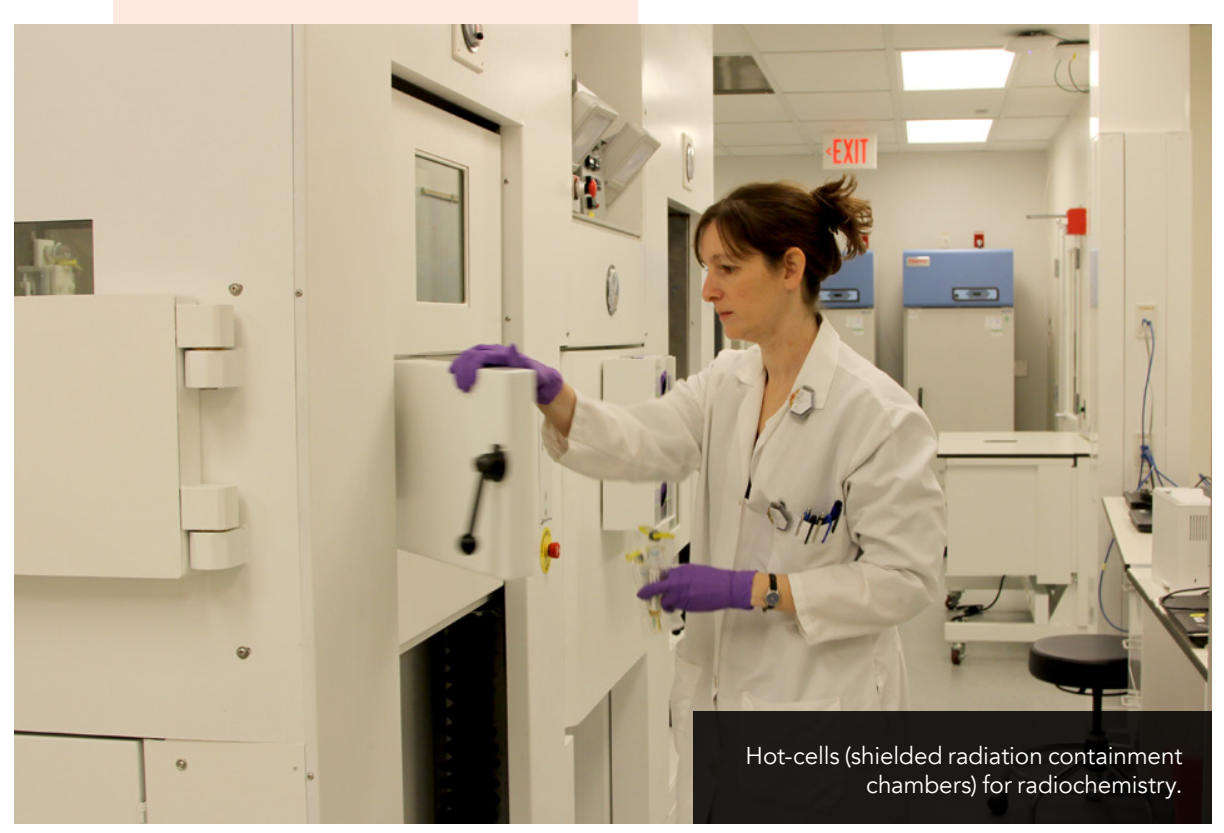

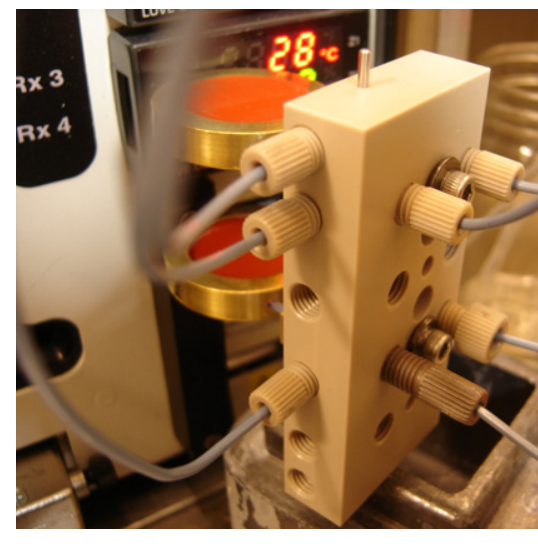

Microreactor for the radiochemical syntt
PET radiotracers and labelling agents.

Some medical imaging techniques make use of radioactive tracers to capture not just static images of the structures in the mody but also to visualse biochemical mediators and processes, and thereby gan emission tomography (PET). This uses radioactive tracers that very quickly. decay to give pairs of gamma rays of a specific energy that can penetrate tissue. These rays can be detected when arriving outside the body by sensitive PET scanners. A scanner surrounds an area of the body (e.g. the trunk or brain) with highly sensitive gamma ray detectors that record images every few minutes. In this manner, the location of the radiotracer in the body, even accurately determined. If you go for Es scan, you will be carefully placed inside the scanner and you will usu
given the radiotracer by injection. kind of imaging technique is positron in an organ such as the brain, can be

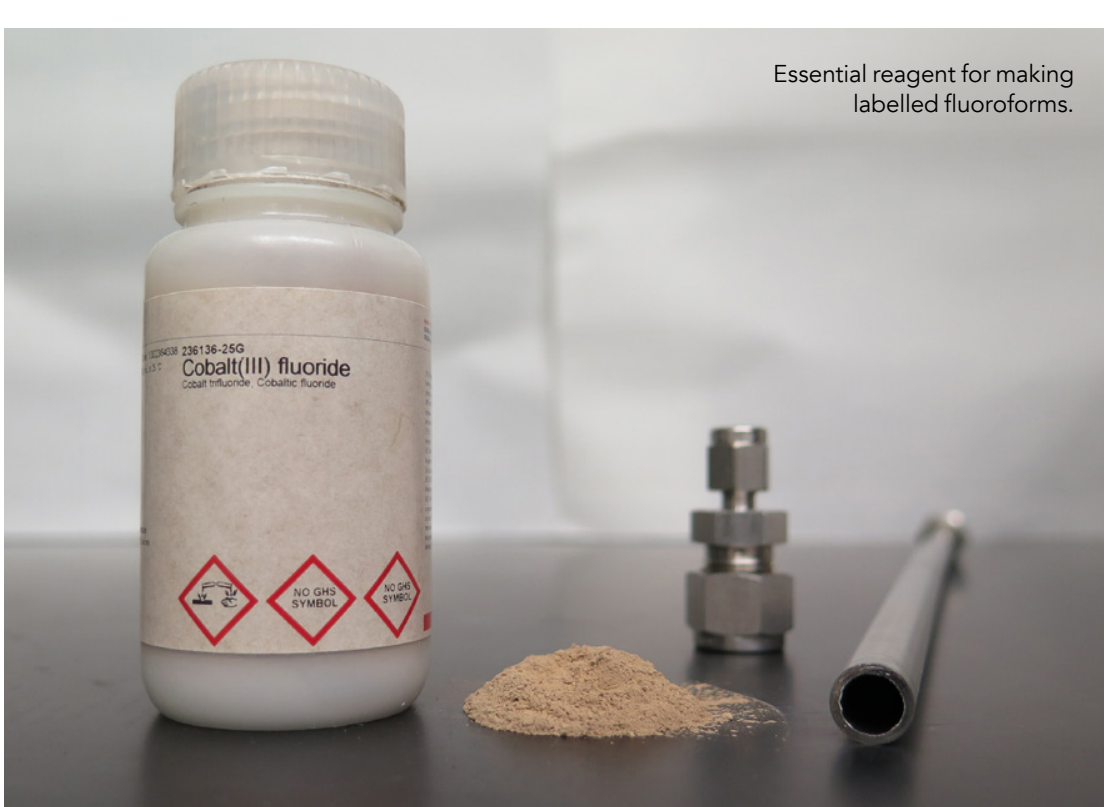

RADIOACTIVE TRACERS The radiotracer is key to providing in disease, including neurological and to understanding disease processes and their treatment. The kind of information that is obtained depends critically on the molecular design of the radiotracer For example, a radiotracer based on glucose, an important energy source that we obtain from food, will be transported to cells that need
glucose for energy. The brain uses glucose for energy. The brain uses glucose almost exclusively for its energy. Cells with a higher energy so greater amounts of gliose, and up in regions with these cells. In this way, for example, energy-demanding tumours may be detected as very bright spots on a PET scan.

Radiotracers must contain a source of radioactivity called a positron-emitter, a special kind of radioisotope that gives off gamma rays. Often, these isotopes have very short half-lives, meaning they undergo radioactive point where they no longer emit radiation. Therefore, $\mathrm{PET}$ scans pose little radiation risk. However, there is a constant need and demand for new used to image different facets of DrVictor Pike, Chief of the PET of the Mocular Imaging Brench at the National Institute of Mental Health in the U.S. and his research team focus on developing new radiotracers that expand the range of processes that PET can be used for imaging. especially in the brain.

NEW TOOLS FOR PET

RADIOTRACER DEVELOPMEN DrPike's research looks at new ways of synthesising radiotracer compounds. incorporating either of two positronencounter carbon mainly in the form decay rapidly, ultimately to the otope. Carbon-11 is still carbon but The makes it unstable. The half-life of carbon-11 is just 20 minutes - mean emains disappears. The positron that is emitted during decay annihilates with a nearby electron to give the two equal energy gamma rays according to Einstein's equation, $E=m c^{2}$.

There is no natural source of carbon-1 or fluorine-18. These radioactive

sotopes must be created with machines known as cyclotrons, where the nuclei of particular types of stable atom are bombarded with high energy particles to make new radioactive atoms. Part of the problem with carbon-11 and from a cyclotron only as pert produced simple chemical compounds like carbo dioxide, methane and fluoride. This means the chemical conversion of one

of these to make a more complex ages of structures in the body but $\begin{aligned} & \text { PET radiotracer is } \\ & \text { challenging and a } \\ & \text { race against time. }\end{aligned}$ PET radiotracer is
challenging and a
race against time. also visualise biochemical mediators and

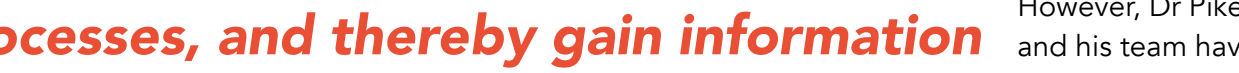
normal and impaired function. found a way of synthesising several new radioactive new chemistry to be developed to reagents, including ["C]fluoroform, successfully incorporate a positron- in which stable carbon is replaced DrVictor Pike, Chief of the PET WT With carbon-11, and / Fffluorofor Typically, radiotracers are made by emitters, carbon-11 or fluorine-18, into the tracer. In our daily lives we and is therefore known as a stable fluoroform in which one nonadioactive fluorine atom is replaced advantage of his synthetic routes to

PET image of OGA enzyme in the human brain. that every 20 minutes half of what of these to make

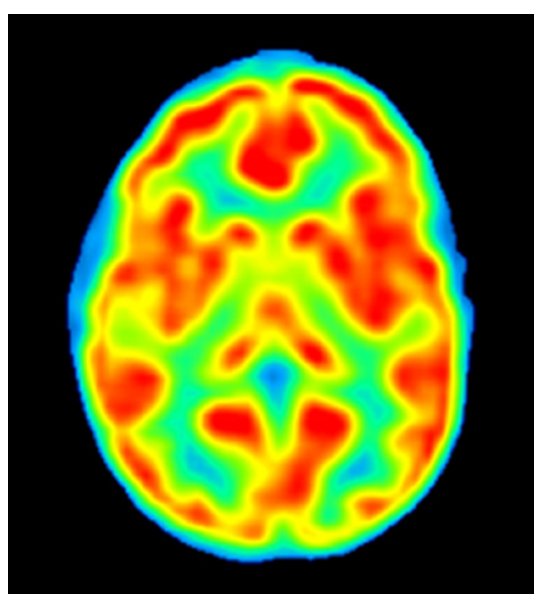




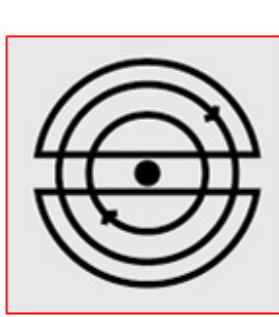

Cyclotron $\frac{{ }^{14} \mathrm{~N}(\mathrm{p}, \alpha)^{11} \mathrm{C}}{\mathrm{N}_{2}-10 \%}$
$\frac{\mathrm{H}_{2}{ }^{18} \mathrm{O}}{{ }^{18} \mathrm{O}(\mathrm{p}, \mathrm{n})^{18} \mathrm{~F}}$

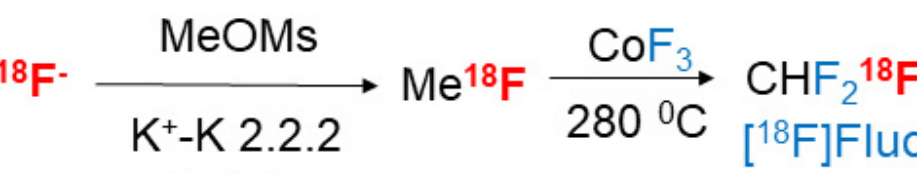

$$
\begin{aligned}
& \text { DMSO }
\end{aligned}
$$$$
130{ }^{\circ} \mathrm{C}, 15 \mathrm{~min}
$$

\section{Syntheses of new, hithy
fluorine-18 ion ( $(\mathrm{B})$.).}

these new radioactive reagents is that they can be performed in the gas phase and are fast, highly reliable, and reproducible. Being able to synthesise more complex radioactive chemicals than carbon dioxide and methane means a greater number of starting materias are avilable for even more radiotracers. The new reagents are highly versatile and efficient in their applications. For [" ${ }^{11} \mathrm{Clfluoroform}$ the synthesis process makes use of cyclotron-made radioactive

["C]methane, and is rapid, simple, and reliable. Another even more recent development makes a usefu radioactive analogue of phosgene from "C-labelled carbon dioxide. This also shows great potential for making new types of PET radiotracer.

\section{NEW POSSIBIITIES}

[" $\left.{ }^{11}\right]$ Fluoroform and other radioactive reagents developed by $\mathrm{Dr}$ Pike and kinds of radiotracer with posibilities to look at a broader range of biological

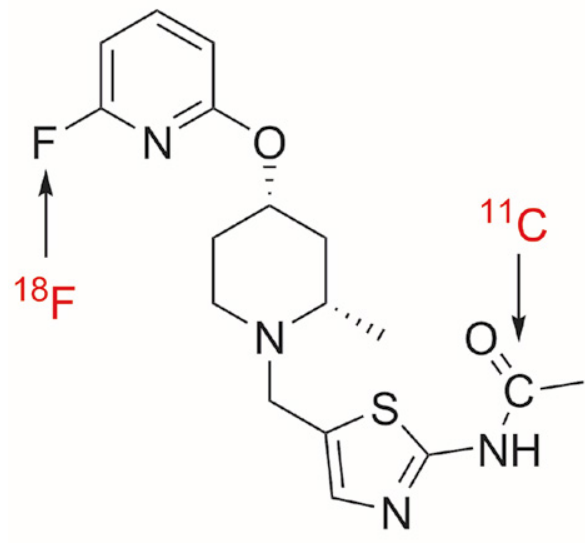

Radiotracer for OGA enzyme imaging showing two possible positron-emitters. The
is a potential rigug target for treating
Alzheimer's disease
One of the enzymes in the brain that has just now been visualised is a potential drug target for Alzheimer's treatments.

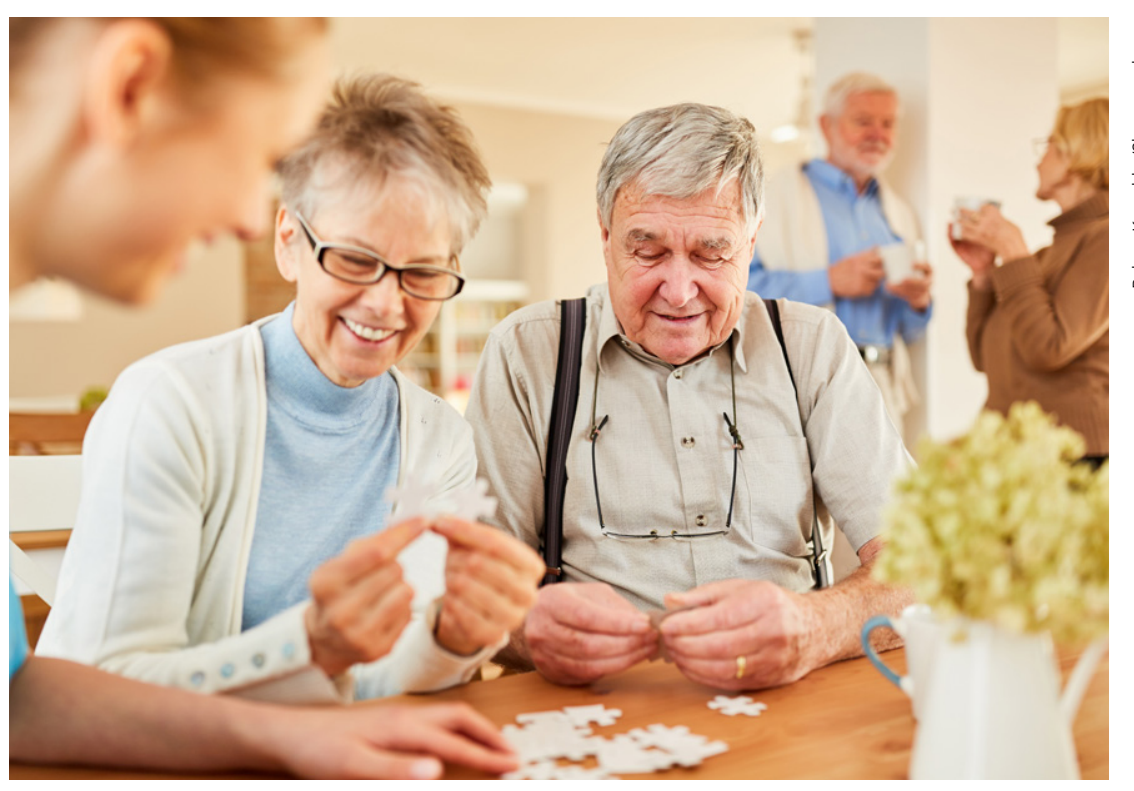

The research team is able to use new kinds of radiotracers to visualise enzymes and proteins in the

processes and their biochemica components. Biochemical components include, for example, enzymes and proteins laid down in Alzheimer's disease, and proteins and enzymes involved in harmful inflammatory in the brain that has just now been visualsed is a potential drug target for Alcheimer's treatments. PET imaging how new candidate drugs might affeet this target, and whether the drugs might work.

The success of PET as a medical imaging technique is dependent on the creation of suitable radiotracers. Creating new compounds, either for radioactive labelling of radiotracers complex process, particularly given fluorine-18 as both isotopes undergo radioactive decay on short timescales. However, Dr Pike's developments possibiea only increase the possibilties of chemical compounds that can be created with these unstable isotopes, but also the possibilities future. This will help to unleash the the short lifetimes of carbon-11 and fo what can be seen with PET in the or to use as radiotracers directly, is technique's potential for medical
Research Objectives

Dr Pike and his team have successfully synthesised new radioactive labelling agents with potential to usher in a new realm of PET radiotracers.

\section{Detail}

PET Radiopharmaceutical Sciences Section

Molecular Imaging Branch

National Institute of Mental Health

Rmational Insti

10 Center Drive

20892-1003, USA

io

Dr Victor W. Pike is a chemist and Chief of the PET maging Branch of the National Institute of Mental Health (USA). He has over four decades of experience eveloping radiotracers for brain imaging with PET.

Funding

The Intramural Research Program of the National Institute of Mental Health (NIMH)

Collaborators

Chers on the papers (Dr Sanjay Telu, Dr Shuiyu Lu, Haskali, Dr Jimmy E. Jakobsson), and Dr Robert B. Innis for PET imaging

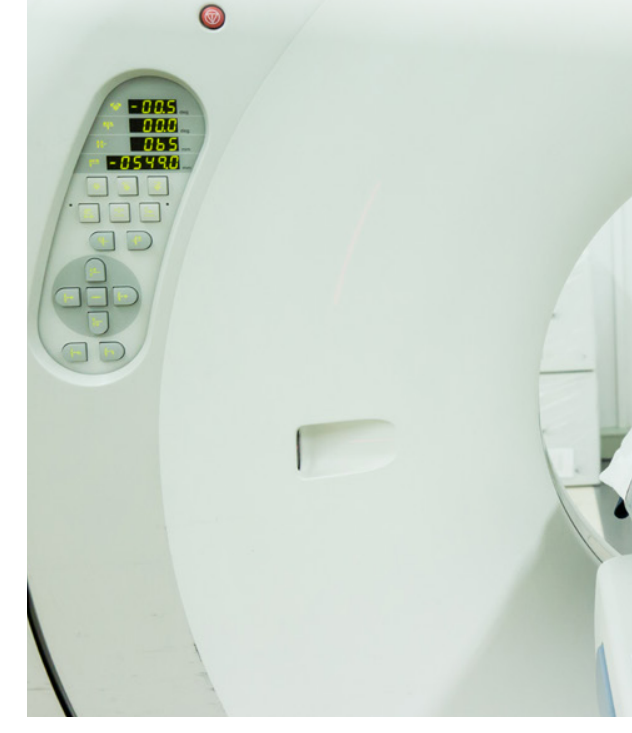

References

Lu, S., Haskali, M.B., Ruley, K.M., Dreyfus, N.J., Dubois, S.L., Paul, S., Pike, V.W. et al. (2020). PET ligands [OH]LSN3316612 and [ CLLSN316612 quantify O-linked$\beta$-N-acetylglucosamine hydrolase in the brain. Science https://doi.org/10.1126/scitranslmed.aau2939

Jakobsson, J.E., Lu, S., Telu, S., and Pike, V.W. (2020). ["C]Carbonyl Difluoride - a New and Highly Efficient International Edition, 59, 7256-7260. Available at: https://doi.org/10.1002/anie.201915414

Yang, B.Y., Telu, S., Haskali, M.B., Morse, C.L., and Pike, V.W. (2019). A Gas Phase Route to ${ }^{18} \mathrm{~F}$ ]fluoroform with Limited Molar Activity Dilution. Scientific Reports, 9, 14835. Haskali M.B. and Pike, VW (2017) ["ClFluoroform, a Breakthrough for Versatile Labeling of PET Radiotracer Trifluoromethyl Groups in High Molar Activity. Chemistry A European Journal, 23, 8156-8160. Avallable at:

https://doi.org/10.1002/chem.201701701

\section{Personal Response}

\section{What do you think are the next major challenges in}

II Aside from the major challenges of discovering everwith carbon-11 or fluorine-18, major challenges still lie ahead in the design of PET radiotracers to show exquisite precision and sensitivity for measuring biological phenomena. This requires strong collaborative efforts.

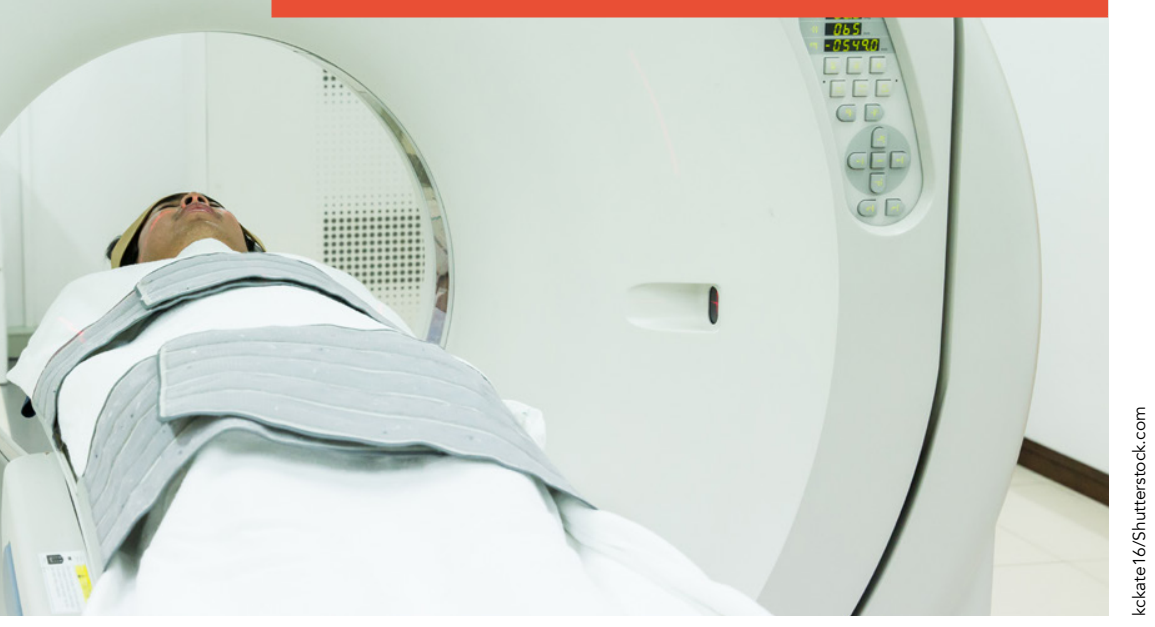

